\title{
INSEPARABLE FINITE SOLVABLE GROUPS. II
}

\author{
HOMER BECHTELL
}

\begin{abstract}
A finite group is called inseparable if the only normal subgroups over which it splits are the group itself and the trivial subgroup. Let $E$ be the formation of finite solvable groups with elementary abelian Sylow subgroups. This note establishes the fact that, up to isomorphism, there is exactly one nonnilpotent inseparable solvable group in which the $E$-residual is a metacyclic p-group.
\end{abstract}

A finite group is called inseparable if the only normal subgroups over which it splits are the one-element subgroup and the group itself (see [1]). These groups are of interest since an arbitrary finite group $G,|G| \neq 1$, is expressible as a product of subgroups, $G=A_{n} A_{n-1} \cdots A_{1}$, for which

(a) $A_{i}$ is inseparable and $\left|A_{i}\right| \neq 1$, for $i=1, \ldots, n$,

(b) $\left(A_{n} \cdots A_{j+1}\right) \triangleleft\left(A_{n} \cdots A_{j}\right)$ for $j=1, \ldots, n-1$, and

(c) $A_{n} \cdots A_{j}=\left[A_{n} \cdots A_{j+1}\right] A_{j}$ for $j=1, \ldots, n-1$.

Previous results [1] suggest that the splitting of a group $G$ over its normal subgroups seems to be determined by the $E$-residual, $G_{E}$, for the formation $E$ of groups having elementary abelian Sylow subgroups. A minimal inseparable nonnilpotent solvable finite group $G$ with $G_{E}$ as a metacyclic $p$-group is known [1] to be isomorphic to the group in the following example.

EXAMPLE. Let $H=\left\langle a, b \mid a^{4}=1, b^{2}=a^{2}, b^{-1} a b=a^{3}\right\rangle$ represent the quaternion group and $K=\left\langle x, y \mid x^{3}=y^{4}=1, y^{-1} x y=x^{2}\right\rangle$. There exists a homomorphism $\theta: K \rightarrow \operatorname{Aut}(H)$ having $\operatorname{Ker}(\theta)=\left\langle y^{2}\right\rangle$ and $K^{\theta}$ isomorphic to the symmetric group of degree 3. Form the partial semidirect product $G$ of $H$ by $K$ such that $H \cap K=\operatorname{Ker}(\theta)=Z(H)$. Then $G$ is inseparable.

The purpose of this note is to establish that each nonnilpotent inseparable finite solvable group $G$ for which $G_{E}$ is a metacyclic p-group is isomorphic to the group in the example.

Only finite solvable groups are to be considered. The notation may be found in [3] with the exception that $G=[A] B$ denotes the splitting of a group $G$ over a normal subgroup $A$ by a subgroup $B$. A group is called a reduced product of a normal subgroup $A$ by a subgroup $B$ if $G=A B$ and $A C$ is a proper subgroup of $G$ for each proper subgroup $C$ of $B$.

Presented to the Society, January 23, 1976; received by the editors February 16, 1976.

AMS (MOS) subject classifications (1970). Primary 20D10, 20 D40.

Key words and phrases. Finite solvable groups, inseparable groups.

- American Mathematical Society 1977 
LEMMA 1. Let $G$ be a nonnilpotent inseparable solvable group in which $G_{E}$ is a metacyclic p-group.

(a) $p=2$.

(b) $\Phi\left(G_{E}\right)=\Phi(G) \subset \Phi(P) \subset G$ for a Sylow 2-subgroup $P$ of $G$.

(c) $G_{E} / \Phi(G)$ is an elementary abelian subgroup of order 4 that is a minimal normal subgroup in $G / \Phi(G)$.

(d) Each normal maximal subgroup of $G$ has index 2 in $G$.

(e) $(G / \Phi(G)) / C_{G / \Phi(G)}\left(G_{E} / \Phi(G)\right) \cong S_{3}, S_{3}$ the symmetric group of degree 3 .

(f) All Sylow q-subgroups of $G$ for $q>3$ are contained in $C_{G}\left(G_{E}\right)$.

Proof. For a group $G$ satisfying the hypothesis, we know from Theorem 3.3 and (3.2) in [1] that $\Phi\left(G_{E}\right)=\Phi(G) \subset \Phi(P) \subset G$ for a Sylow p-subgroup $P$ of $G, G_{E} / \Phi(G)$ is an elementary abelian subgroup of order $p^{2}$ that is a minimal normal subgroup in $G / \Phi(G)$, and each normal maximal subgroup of $G$ has index $p$ in $G$. Consequently, (b), (c), and (d) are restatements for $p=2$. So consider (a). The group $H=G / \Phi(G)$ satisfies the conditions of $\Phi(H)=1$, $H_{E}=(G / \Phi(G))_{E}=G_{E} / \Phi(G)$ is a minimal normal subgroup of order $p^{2}$ in $H$, $1 \subset \Phi\left(P^{*}\right) \subset H_{E}$ for a Sylow $p$-subgroup $P^{*}$ of $H$, and each normal maximal subgroup has index $p$ in $H$. Moreover $H^{\prime}$ is the intersection of the normal maximal subgroups of $H, H / H^{\prime}$ is an elementary abelian $p$-group, and each Sylow $q$-subgroup is contained in $H^{\prime}$ for $q \neq p$.

Clearly $H / C_{H}\left(H_{E}\right) \cong S \subseteq \mathrm{GL}(2, p)$. If $S$ was abelian, then $H^{\prime} \subseteq C_{H}\left(H_{E}\right)$. Consequently $|S|$ would be a power of $p$ and $C_{H}\left(H_{E}\right)$ would contain the Sylow $q$-subgroups of $H$ for $q \neq p$. Since $|\mathrm{GL}(2, p)|=p(p-1)^{2}(p+1)$, then $|S|$ $=1$ or $p$. But $\left|\Phi\left(P^{*}\right)\right|=p$. So in either case, $\Phi\left(P^{*}\right) \subseteq Z(H)$. This contradicts the irreducibility of $H_{E}$. Therefore $S$ is nonabelian. Moreover $S=\left[S^{\prime}\right] A$ with $|A|=p$, each Sylow subgroup of $S$ is elementary abelian (since $S \in E$ ), and each Sylow $q$-subgroup is contained in $S^{\prime}$ for $q \neq p$.

Since $\operatorname{GL}(2, p)=[\operatorname{SL}(2, p)] K^{*}$ and $\left|K^{*}\right|=p-1$, then $S \subseteq \operatorname{SL}(2, p)$. For $p=2, \operatorname{SL}(2,2) \cong S_{3}, S_{3}$ the symmetric group of degree three. The structure of $S$ implies that $S \cong S_{3}$. Consider $p>2$. It is known (see [3, p. 196]) that the Sylow $q$-subgroups of $\operatorname{SL}(2, p)$ are cyclic for $q \neq 2$ and generalized quaternion for $q=2 \neq p$. Therefore $S$ is a nonabelian group of square-free order. So $S^{\prime} \subseteq F(S)$. But $S$ is not nilpotent. Hence $S^{\prime}=F(S)$. However $\Phi(S)=1$ implies that $S^{\prime}$ is cyclic and completely reducible. Consequently $A$ acts faithfully on each Sylow $q$-subgroup in $S^{\prime}$. Hence $p \mid(q-1)$ for each prime $q \neq p$. This implies that either $p=q-1$ or $p<q-1$. Since each prime $q \neq p$ also must satisfy the condition that $q$ divides $p(p-1)^{2}(p+1)$, then $q=p+1$ or $q<p$. The only possible primes that can be considered are those for which $q=p+1$. Therefore $p=2, q=3$, and $S \cong S_{3}$. Hence both (a) and (e) are valid.

From the above, it follows that for each prime $q>3$, the elements in a Sylow $q$-subgroup of $H$ must centralize $H_{E}=G_{E} / \Phi(G)$. Since $\Phi(G)=$ $\Phi\left(G_{E}\right)$, then these elements centralize $G_{E}$ by Burnside's result (see [2, p. 174]). This verifies (f). 
LEMMA 2. If $G_{E}$ is a metacyclic 2-group and $G$ is a nonnilpotent inseparable solvable group, then $G_{E}$ is either a homocyclic 2-group or a quaternion group.

Proof. By Lemma 1(e), there exists an inner automorphism $\alpha$ of order 3 that acts irreducibly and faithfully on the elementary abelian 2-group $G_{E} / \Phi\left(G_{E}\right)$ of order 4 . If $G_{E} / G_{E}^{\prime}$ is decomposable with respect to $\alpha$, then $G_{E} / \Phi\left(G_{E}\right)$ is reducible. Hence $G_{E} / G_{E}^{\prime}$ is indecomposable with respect to $\alpha$.

If $G_{E}^{\prime}=1$, then $G_{E}$ is a homocyclic abelian 2-group (see [2, p. 176]).

Assume that $G_{E}^{\prime} \neq 1$. Then $G_{E} / G_{E}^{\prime}$ is a homocyclic abelian 2-group. Since $G_{E}^{\prime}$ is cyclic, there exists a chief factor $G_{E}^{\prime} / N$ of order 2. Let $M / G_{E}^{\prime}$ $=\Omega_{1}\left(G_{E} / G_{E}^{\prime}\right)$. Then $M \triangleleft G$ and $M / G_{E}^{\prime}$ is elementary abelian of order 4. So $|M / N|=8, \Phi(M / N)=G_{E}^{\prime} / N$, and $\alpha$ acts irreducibly on $M / G_{E}^{\prime}$. If $M / N$ is abelian, then it is of type $\left(2^{2}, 2\right)$. However $\Omega_{1}(M / N)$ has order 4 and it is characteristic in $M / N$. This contradicts the fact that $M / G_{E}^{\prime}$ is irreducible. Consequently $M / N$ is nonabelian of order 8 and $G_{E}^{\prime} / N=\Phi(M / N)$ $=(M / N)^{\prime}$. Since the automorphism group of a dihedral group does not contain an element of order 3 , then $M / N$ must be a quaternion group. Because $G_{E} / G_{E}^{\prime}$ is homocyclic, then either $M=G_{E}$ or $M / G_{E}^{\prime} \subseteq \Phi\left(G_{E} / G_{E}^{\prime}\right)$. The second case implies that $M / N \subseteq \Phi\left(G_{E} / N\right)$. However a subgroup normal in a group and contained in the Frattini subgroup cannot be isomorphic to a quaternion group by results of Gaschütz (see [3, p. 272]). Hence $M=G_{E}$ and $\Phi\left(G_{E}\right)=G_{E}^{\prime}$. By a result of O. Taussky (see [3, p. 339]), $G_{E}$ is isomorphic to one of the following 2-groups: dihedral, quasi-dihedral, or generalized quaternion. The only possibility that would admit $G_{E} / N$ as a quaternion group is that $G_{E}$ itself is a quaternion group.

THEOREM. Let $G$ be a nonnilpotent solvable group that has $G_{E}$ as a nonabelian metacyclic p-group. If $G$ is inseparable, then $G$ is isomorphic with the group of the Example.

Proof. From Lemma $1, G_{E}$ is a 2-group and $G_{E} / \Phi(G)$ is a minimal normal subgroup of $G / \Phi(G)$. Then

$$
F(G / \Phi(G))=F(G) / \Phi(G)=G_{E} / \Phi(G) \otimes C / \Phi(G)
$$

for a subgroup $C \triangleleft G$ by a result of Gaschütz [3, p. 279]. Furthermore $G / \Phi(G)=[C / \Phi(G)](B / \Phi(G))$ for a subgroup $B$ in $G$ by Hilfsatz 4.4 [3, p. 278]. Since $\Phi(G)$ is a 2-group and $G$ is inseparable, then $F(G)$ cannot have a direct $q$-factor for $q \neq 2$. So $F(G)$ is a 2-group. For each Sylow 2-subgroup $P$ of $G,[P, C] \subseteq G_{E} \cap C=\Phi(G)$. Hence $P / \Phi(G) \subseteq C_{G / \Phi(G)}(C / \Phi(G))=$ $D$. By Lemma 1(d), each normal maximal subgroup of $G$ has index 2 . Since $D$ $\triangleleft G / \Phi(G)$ and $(G / \Phi(G): D)$ is prime to 2 , then a contradiction arises unless $D=G / \Phi(G)$. Consequently $C / \Phi(G) \subseteq Z(G / \Phi(G))$. So $B \triangleleft G$. Since 
$F(G / \Phi(G))=G_{E} / \Phi(G) \otimes C / \Phi(G)$, then each element that centralizes $G_{E} / \Phi(G)$ also must centralize $F(G) / \Phi(G)$. Hence

$F(G) / \Phi(G) \subseteq C_{G / \Phi(G)}\left(G_{E} / \Phi(G)\right) \subseteq C_{G / \Phi(G)}(F(G) / \Phi(G))=F(G) / \Phi(G)$.

Therefore

$$
C_{G / \Phi(G)}\left(G_{E} / \Phi(G)\right)=F(G) / \Phi(G)=C G_{E} / \Phi(G) .
$$

By Lemma $1(\mathrm{e}), G / C G_{E} \cong S_{3}$.

From Lemma 2, $G_{E}$ is either a homocyclic abelian 2-group or a quaternion group. Let $G=C B$ be the decomposition given above and suppose that $G_{E}$ is a homocyclic abelian 2-group. There exists a reduced product $G=\left(C B_{E}\right) A$ for a subgroup $A \subseteq B$. From the definition of a reduced product, it is evident that $C B_{E} \cap A \subseteq \Phi(A) \neq 1$. Since $E$ is a formation, $A_{E} \subseteq B_{E}$. Then $C B_{E}$ $\cap A \subseteq \Phi(A) \subseteq A_{E} \subseteq C B_{E} \cap A$. Hence $C B_{E} \cap A=\Phi(A)=A_{E}$. Furthermore $C G_{E} \subseteq C B_{E} \subseteq C G_{E}$, since $G / C B_{E} \in E$. Therefore $C B_{E}=C G_{E}$. So

$$
S_{3} \cong G / C G_{E}=G / C B_{E} \cong A /\left(C B_{E} \cap A\right)=A / A_{E}=A / \Phi(A) .
$$

Consider $A / \Phi^{2}(A)$. Since $\Phi(A)$ is metacyclic, then $\left|\Phi(A) / \Phi^{2}(A)\right|=4$ or 2. If $\left|\Phi(A) / \Phi^{2}(A)\right|=4$, then $\left|A / \Phi^{2}(A)\right|=24$. But a group of that order with $\left(A / \Phi^{2}(A)\right)_{E}=A_{E} / \Phi^{2}(A)$ being elementary abelian of order 4 and $\left(A / \Phi^{2}(A)\right) /\left(A / \Phi^{2}(A)\right)_{E} \cong A / A_{E} \cong S_{3}$ has a Frattini subgroup of order 1 or 2 . Since $\Phi(A) \neq 1$, then $\left|\Phi(A) / \Phi^{2}(A)\right|=2$. Each element of order 3 in $A$ induces by conjugation an automorphism $\alpha$ that fixes $\Phi(A) / \Phi^{2}(A)$ pointwise. By Burnside's result [2, p. 174], $\Phi(A)$ is a set of fixed points for $\alpha$. Since $G_{E}$ is homocyclic and $\alpha$ acts irreducibly on $G_{E} / \Phi\left(G_{E}\right)$ by Lemma $1(\mathrm{c})$, then $\alpha$ acts irreducibly on $\Omega_{1}\left(G_{E}\right)$. A contradiction arises since $\Omega_{1}(\Phi(A)) \subset \Omega_{1}\left(G_{E}\right)$. Therefore $G_{E}$ cannot be a homocyclic abelian 2-group.

Next suppose that $G_{E}$ is a quaternion group. Then $C \cap B=\Phi(G)=$ $\Phi\left(G_{E}\right)$ by Lemma $1(\mathrm{~b})$. Since $G / C B_{E} \in E, G_{E} \subseteq C B_{E}$. So

$$
G_{E}=G_{E} \cap C B_{E}=B_{E}\left(G_{E} \cap C\right)=B_{E} \Phi(G)=B_{E} \Phi\left(G_{E}\right)=B_{E} .
$$

Hence

$$
\begin{aligned}
S_{3} & \cong G / C G_{E}=G / C B_{E} \cong B /\left(C B_{E} \cap B\right) \\
& =B / B_{E}(C \cap B)=B_{E} \Phi\left(G_{E}\right)=B / B_{E} .
\end{aligned}
$$

Assume that $C \neq \Phi(G)$. Since $C / \Phi(G) \subseteq Z(G / \Phi(G))$, then $C / \Phi(G)$ contains a subgroup $N / \Phi(G)$ of order 2 that is a minimal normal subgroup of $G / \Phi(G)$. By Hilfsatz $4.4[3$, p. 278], there exists a normal subgroup $M / \Phi(G)$ such that $G / \Phi(G)=N / \Phi(G) \otimes M / \Phi(G)$. The order of $N$ is 4 and $N$ must be cyclic for otherwise $G$ splits over $M$. Since $\left(G: C_{G}(N)\right) \leqslant 2$ and $M$ is a normal maximal subgroup in $G$ of index 2 , then $G_{E} \subseteq M \cap C_{G}(N)$. Let $N_{1}$ be a maximal subgroup of $G_{E}$. Then $N N_{1}$ is an abelian subgroup of order 8 that has 
at least two cyclic subgroups of order 4. So $\left|\Omega_{1}\left(N N_{1}\right)\right|=4$. If $\Omega_{1}\left(N N_{1}\right) \nsubseteq M$, then $G$ splits over $M$ and a contradiction arises. Since $N_{1} \subset G_{E} \subset M$, then $\Omega_{1}\left(N N_{1}\right) \subset M$ implies that $N \subset N N_{1}=N_{1}\left(\Omega_{1}\left(N N_{1}\right)\right) \subset M$. This second contradiction implies that $C=\Phi(G)$. Consequently $G=B$. From the results in [1], this group is isomorphic to the group in the Example.

A comment is in order. The result here complements [1]; the choice of structure for $G_{E}$ was not arbitrarily selected.

\section{BIBLIOGRAPHY}

1. H. Bechtell, Inseparable finite solvable groups, Trans. Amer. Math. Soc. 216 (1976), 47-60.

2. D. Gorenstein, Finite groups, Harper and Row, New York, 1968. MR 38 \#229.

3. B. Huppert, Endliche Gruppen. I, Springer-Verlag, Berlin, 1967. MR 37 \#302.

Department of Mathematics, University of New Hampshire, Durham, New Hampshire 03824 